EPJ Web of Conferences 107, 03010 (2016)

DOI: $10.1051 /$ epjconf/201610703010

(C) Owned by the authors, published by EDP Sciences, 2016

\title{
Phases and phase transitions in the algebraic microscopic shell model
}

\author{
A. I. Georgieva ${ }^{1, a}$ and K. P. Drumev ${ }^{2}$ \\ ${ }^{1}$ Institute of Solid State Physics, Bulgarian Academy of Sciences, Sofia 1784, Bulgaria \\ ${ }^{2}$ Institute for Nuclear Research and Nuclear Energy, Bulgarian Academy of Sciences, Sofia 1784, Bulgaria
}

\begin{abstract}
We explore the dynamical symmetries of the shell model number conserving algebra, which define three types of pairing and quadrupole phases, with the aim to obtain the prevailing phase or phase transition for the real nuclear systems in a single shell. This is achieved by establishing a correspondence between each of the pairing bases with the Elliott's SU(3) basis that describes collective rotation of nuclear systems. This allows for a complete classification of the basis states of different number of particles in all the limiting cases. The probability distribution of the SU(3) basis states within theirs corresponding pairing states is also obtained. The relative strengths of dynamically symmetric quadrupole-quadrupole interaction in respect to the isoscalar, isovector and total pairing interactions define a control parameter, which estimates the importance of each term of the Hamiltonian in the correct reproduction of the experimental data for the considered nuclei.
\end{abstract}

\section{Introduction}

The present-day microscopic approaches for the description of the complex nuclear spectra are based either on mean-field methods or on shell model [1]. Both are employing complicated computer intensive algorithms and consequently are rather cumbersome, time consuming and difficult for direct application to the interpretation of the experimental results.

In such cases it is most reasonable to introduce symmetry ruled limiting cases or dynamical symmetries, that are exactly solvable and can serve as reference cases for specific nuclear properties. In this paper we outline such dynamical symmetries that can be identified in the number conserving shell model algebra. They introduce the two most important residual interactions of the many-body nuclear system - the pairing and quadrupole-quadrupole interaction, which are the fundamental terms in the microscopic version of the Pairing plus Quadrupole Model (PQM) [2-4]. Of cause these limiting cases are rather schematic and describe the spectra of a restricted number of real nuclei, but they can be considered as different phases of the complex interactions acting, between the nuclear constituents. Consequently it is our further aim to establish possible relations between the subalgebras in the chains of the dynamical symmetries and to employ them in evaluating the importance of each interaction in the formation of the nuclear spectra.

In this talk we shortly outline the theoretical background of our approach. First we identify the reduction schemes of the shell model number-conserving algebra $U(\Omega)$, which are governed by the Wigner's supermultiplet model (the LST-scheme) [5], and practically separates the

\footnotetext{
ae-mail: anageorg@issp.bas.bg
}

shell-model space to spatial and spin-isospin part. Then, we investigate the dynamical symmetries that reduce the spatial part to the angular momentum $L$ algebra $S O(3)$. These reductions are predetermined by the residual interactions, which we would like to consider, namely the pairing and quadrupole-quadrupole ones. As a result, we establish a correspondence between some of the subgroups in the reduction chains that define the considered dynamical symmetries, which allows for the investigation of the competing and complementarity features of the considered interactions. The later is achieved by evaluation of the control parameter defining the phase transitions between the considered symmetries. These results are obtained in the applications of the theory, which are presented in an separate contribution to the proceedings.

\section{Reduction schemes in the microscopic shell model}

The many-particle shell model wave functions are constructed by filling the single-particle orbitals of the valence shells with nucleons, taking into account the Pauli principle [6]. As a result an antisymmetrization of the total wave function, containing the product of the spatial, spin and isospin parts, is required as dictated by the LST coupling scheme. Consequently, the antisymmetric irreducible representations of $U(4 \Omega)$ for $m$ - particles, labeled by the Young diagrams $\left[1^{m}\right]$ is reduced in the following way:

$$
\begin{gathered}
U(4 \Omega) \supset U_{S T}(4) \otimes U(\Omega) \\
{\left[1^{m}\right] \quad\{\tilde{f}\} \quad\{f\}}
\end{gathered}
$$

The labels of the respective representations are given below the group symbols. Further, according to the Wigner's 
supermultiplet model $[6,7]$, the reduction

$$
\begin{array}{ccl}
S U_{S T}(4) \supset & S U_{S}(2) \otimes & S U_{T}(2), \\
\{\tilde{f}\} & S & T
\end{array}
$$

gives the total spin $S=\sum_{i} s_{i}$ and isospin $T=\sum_{i} t_{i}(i$ enumerates the considered particles) values of the states wave functions, which together with the orbital angular momentum $L=\sum_{i} l_{i}$ of $S O_{L}(3)$ are good quantum numbers in this case. Further, $L$ and $S$ are coupled to total angular momentum $\mathbf{J}=\mathbf{L}+\mathbf{S}$. Using the isomorphism of the algebras $S U_{S T}(4) \sim S O_{S T}(6), S U_{S}(2) \sim S O_{S}(3)$ and $S U_{T}(2) \sim S O_{T}(3)$, another shell-model-reduction chain equivalent to the chain (2) can be identified [8]

$$
\begin{array}{ccc}
S U_{S T}(6) \supset & S O_{S}(3) \otimes & S O_{T}(3) \\
{\left[P_{1}, P_{2}, P_{3}\right]} & S & T
\end{array}
$$

\subsection{Reductions of the spatial part}

While in this way the spin $S$ and the isospin $T$ of the system are specified, there is no general rule for obtaining the values of the orbital angular momentum $L$, contained in the irreps $\{f\}$, since the reduction $U(\Omega) \supset S O_{L}(3)$ is not a canonical one.

Elliott's $S U$ (3) model [9] provides an elegant and analytically solvable way for obtaining the missing labels in the reduction of the spatial part to the orbital angular momentum $L$, by introducing the reduction [10]

$$
\begin{array}{llll}
U(\Omega) \supset & S U(3) \supset & S O(3) \\
\{f\} \quad \alpha & (\lambda, \mu) & K L
\end{array}
$$

where $\alpha$ indicates the multiplicity of the $S U(3)$ representation $(\lambda, \mu)$ in the $U(\Omega)$ representation $\{f\}$. Elliott's model presupposes that the residual interaction has a quadrupole character and can be written as

$$
V_{r o t}=H_{0}+\frac{1}{2} \chi Q \cdot Q
$$

where $Q \cdot Q=4 C_{S U(3)}^{2}-3 L^{2}$ and the eigenvalue of the second invariant of $S U(3)$ is $C_{S U(3)}^{2}=\lambda^{2}+\lambda \mu+\mu^{2}+3(\lambda+\mu)$. Obviously (5) gives rise to a rotational spectra of the type $L(L+1)$. In this way the rotational states are labeled by the quantum numbers of the representations of the algebras in the chain (4):

$$
\left|\Psi_{R}\right\rangle \equiv|\{f\}, \alpha(\lambda, \mu) K L M\rangle \equiv|m, \alpha(\lambda, \mu) K L M\rangle .
$$

Since the microscopic $S U(3)$ model based on the three dimensional harmonic oscillator has a well developed theory, including the Wigner-Racah algebra for the calculation of matrix elements $[11,12]$ in the $S U(3)$-basis and various successful applications in real nuclei [13], we choose to investigate the short range pairing interactions in conjunction with the quadrupole-quadrupole long range interaction introduced by (5). For this purpose we need to introduce in the shell model space dynamical symmetries that account for the pairing correlations in the nuclear structure. In general the eigenvalue problem for the pairing interaction can be solved analytically $[14,15]$ for the three dynamical symmetries contained in the quasispin $S O(8)$ algebra

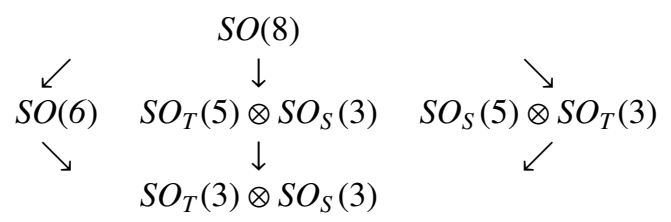

which are used to obtain analytical solutions for the total pairing $(P t)$ interaction containing isoscalar and isovector pairing with equal strengths from the first chain on the left. The middle and righthand side chains of (7) lead to exact solutions for the isoscalar (Pisc) or isovector (Piv) pairings correspondingly. Hence these tree cases are determined by the following three types of residual interactions:

$$
V_{P t}=\mathbf{G}\left\{S_{\mu}^{\dagger} \cdot S_{\mu}+P_{\mu}^{\dagger} \cdot P_{\mu}\right\}
$$

for "total" pairing;

$$
V_{P i s c} \equiv V_{0}=\mathbf{G}_{0} S_{\mu}^{\dagger} \cdot S_{\mu}
$$

for isoscalar pairing and

$$
V_{P i v} \equiv V_{1}=\mathbf{G}_{1} P_{\mu}^{\dagger} \cdot P_{\mu}
$$

for the isovector pairing. The above interactions (8), (9) and (10) are expressed in terms of the isoscalar

$$
S_{\mu}^{\dagger}=\sum_{l} \beta_{l} \sqrt{\frac{2 l+1}{2}}\left(a_{l \frac{1}{2} \frac{1}{2}}^{\dagger} \times \tilde{a}_{l \frac{1}{2} \frac{1}{2}}^{\dagger}\right)_{(0 \mu 0)}^{(010)}
$$

and isovector

$$
P_{\mu}^{\dagger}=\sum_{l} \beta_{l} \sqrt{\frac{2 l+1}{2}}\left(a_{l \frac{1}{2} \frac{1}{2}}^{\dagger} \times \tilde{a}_{l \frac{1}{2} \frac{1}{2}}^{\dagger}\right)_{(00 \mu)}^{(001)},
$$

microscopic tensor pairing operators, since the operators $a_{l \frac{1}{2} \frac{1}{2}}^{\dagger}\left(a_{l \frac{1}{2} \frac{1}{2}}\right)$ are creation (annihilation) operators of a nucleon on an orbit $l$, with $\operatorname{spin} s=1 / 2$ and isospin $t=1 / 2$. The later are part of the generators of the $S O(\Omega)$ and the $S O(8)$ algebras and their second order Casimir invariants are related. Obviously both algebras are part of the reduction of the spatial part $U(4 \Omega)$ to the $S O(3)$ algebra of the angular momentum $L$. Something more they are complementary in the sense of Moshinsky and Quesne [16], as established in [17]. Because of this their representations are labeled by related quantum numbers: $v,\left[p_{1}, p_{2}, p_{3}\right]$ or $[\mu]=\left[\mu_{1}, \mu_{2}, \mu_{3}, \mu_{4}\right]$ with $\mu_{1} \geq \mu_{2} \geq \mu_{3} \geq \mu_{4} \geq 0$, where $v=\sum_{i} \mu_{i}$ is the seniority quantum number for $S O(\Omega)$ and $S O(8)$. Using the complementarity of $U(\Omega)$ and $S U_{S T}(4) \sim S O_{S T}(6)(1),(3)$ and $S O(\Omega)$ and $S O(8)$ the basis states in the reduction to the angular momentum subgroup $\mathrm{SO}_{L}(3)$ could be labeled as

$$
\left|\Psi_{P t}\right\rangle \equiv\left|\{f\} \equiv m, v,\left[p_{1}, p_{2}, p_{3}\right], \beta L M ; S T\right\rangle,
$$

where $\beta$ gives the multiplicity with which the values of the angular momentum $L$ appear in the $S O(8)$ irreducible representations (irreps) $v,\left[p_{1}, p_{2}, p_{3}\right] \equiv v[p]$. Further, the 
reduction $U(\Omega) \supset S O(\Omega) \supset S O_{L}(3)$ can easily be realized using the basic assumption that the fundamental representation $\{1\}_{U(\Omega)}$ is composed by the representations $\left(l_{1}, l_{2}, \ldots l_{r}\right)$ of $S O_{L}(3)$ for nucleons occupying the orbits $l_{1}, l_{2}, \ldots l_{r}$.

Obviously the interaction (8) is diagonal in the basis (13) with eigenvalues given in [17] as well as the reduction rules for obtaining the quantum numbers of the labeling irreps.

Now, we will present another two chains of the number conserving shell model within $U(4 \Omega)$ that correspond to the middle and right chains in (7) describing the pure isoscalar (9) and isovector (10) pairing. As shown in [17], since in these cases one has to start with the $U(4 \Omega) \supset$ $U(2 \Omega) \otimes S U_{\sigma}(2)$ and $U(2 \Omega)$ also contains $S O(\Omega) \otimes S U_{\sigma}(2)$, where $\sigma=S \vee T$, for completing the group chain the algebra $S p(2 \Omega)$ has to be included in $U(2 \Omega)$ and then reduced to $S O(\Omega) \otimes S U_{\sigma}(2)$. Therefore the group-subgroup chain equivalent to the middle and right chains in (7) and contained in the number preserving shell model symmetry algebra $U(4 \Omega)$ are

$$
\begin{array}{llcc}
{[U(2 \Omega) \supset} & S p(2 \Omega) \supset & \left.S O(\Omega) \otimes S U_{S}(2)\right] \\
\left\{f_{s}\right\} & \left\langle\widetilde{\mu_{S}}\right\rangle ; v_{S} t_{S} & v[p] & S .
\end{array}
$$

and

$$
\begin{aligned}
& {\left[U(2 \Omega) \supset \quad S p(2 \Omega) \supset \quad S O(\Omega) \otimes S U_{T}(2)\right]} \\
& \left\{f_{s}\right\} \quad\left\langle\widetilde{\mu_{T}}\right\rangle ; v_{T} t_{T} \quad v[p] \quad T .
\end{aligned}
$$

Respectively each of the algebras in (14) and (15) is in direct product with the $S U_{T}(2)$ for the isoscalar and $S U_{S}(2)$ for the isovector pairing respectively. The quantum numbers given below the subgroup of the chain (14) and (15) are easy to be obtained from the representation $\left\{1^{m}\right\}$ of $U(4 \Omega)$ at fixed $T=0$ or $S=0$ with the help of the reduction rules, based on the pletism and given in [17]. The equivalence of the reduction chains (14) and (15) and the middle and right chains in (7) describing the pure isoscalar (9) and isovector (10) pairing respectively, as established in [17] is due to the complementarity [16] in this case of $S p(2 \Omega)$ to $S O(8)$ and $S O(\Omega) \otimes S U_{\sigma}(2)$ to $\left.S O(5) \otimes S O_{\sigma}(3)\right]$. Than the basis states for the Hamiltonians $V_{0}(9)$ and $V_{1}$ (10) in the reductions (14) and (15) to the angular momentum algebra $S O(3)$ are labeled as

$$
\left|\Psi_{P i s c}\right\rangle \equiv\left|m, S, v_{S}, t_{S}, T, v,[p] \beta L M\right\rangle,
$$

and

$$
\left|\Psi_{P i v}\right\rangle \equiv\left|m, T, v_{T}, t_{T}, S, v,[p] \beta L M\right\rangle,
$$

and the interactions given by (9) and (10) are diagonal in the isoscalar (16) and isovector (17) pairing bases with eigenvalues given in [17]. Therefore, the isovector chain (15) generates rotations $T(T+1)$ in the isospin space and in this way differs from the chain (14). Due to relations between the Casimir invariants of the subgroups in the chains (14), (15) [17] the eigenenergies of (9) and (10) do not explicitly depend on the quantum numbers $v$, [p] of the representations $S O(\Omega) \sim S O(8)$, but instead depend on the quantum numbers $v_{S}, t_{S}$ and $v_{T}, t_{T}$ respectively, which define the representations of $\operatorname{Sp}(2 \Omega) \supset S O(\Omega)$ in each of the considered cases. Then by using the standard methods for the decomposition $O(n) \supset O(n-1)$ [18] one obtains the values of the angular momentum operators $L$ and their multiplicity $\beta$. Hence this reduction explicitly depends on the the $l$ - orbits appropriate for the nucleus under consideration and could be applied in one or several orbits.

\subsection{Unified reductions schemes of shell-model $U(4 \Omega)$ algebra}

Summarizing the reductions of the shell model algebra $U(4 \Omega)$ (1) into the spatial and spin-isospin branches and their complementarity and taking into account that the reduction of the spatial part $U(\Omega)$ to the $S O(3)$ algebra of the angular momentum can be realized in addition to the one through the $S U(3)$ algebra (4) [6] introducing rotations, in three other ways introducing the three types of pairing interactions (8), (9) and (10) all complementary to the reduction of the Wigner's supermultiplet model (2) [5], we can unify the considered above chains into a generalized reduction schemes. Each of them introduces one of the pairing chains, corresponding to the to the tree limiting cases of the $S O(8)$ pairing algebra (7) in conjunction with the chain (4), since we would like to consider the influence of the pairing interactions on the development of the collective rotational spectrum in the real nuclear systems. The first case that we considered in [19] is related to the introduction of the total pairing interaction (8)and is presented in Fig. 1.

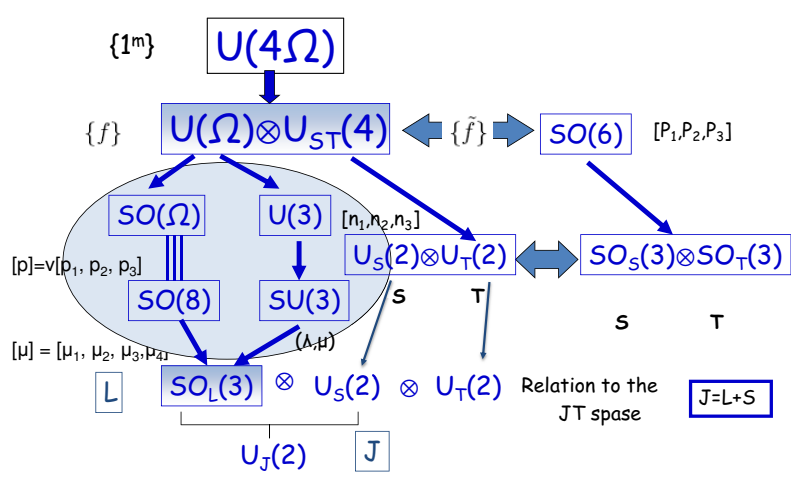

Figure 1. (Color online) Unified reduction scheme for the total pairing chain and the $S U(3)$ chain.

The next scheme presents the case of considering the pure isoscalar pairing obtained through the chain (14) that includes the symplectic algebra $\operatorname{Sp}(2 \Omega)$ and then going down to angular momentum algebra trough the direct product $S O(\Omega) \otimes S U_{S}(2)$. It is presented in Fig. 2. Analogical scheme is obtained for the isovector case by exchange of the labels $S$ and $T$ in the respective $S U(2)-$ algebras and their representations.

In this way we obtain the important result that the left branches starting with $U(\Omega)$ (Fig.1), $U(2 \Omega) \otimes S U_{\sigma}(2)$ (Fig.2) of the shell-model algebra $U(4 \Omega)$ contain two 


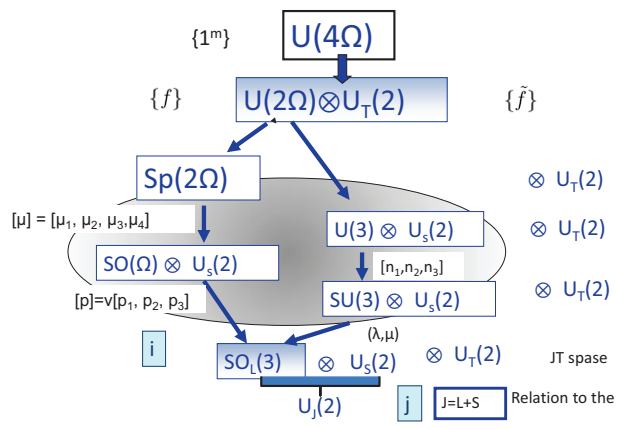

Figure 2. (Color online) Unified reduction scheme for the isoscalar pairing chain and the $S U(3)$ chain.

distinct dynamical symmetries defined by the reduction chains of the pairing interactions and middle branch corresponding to the quadrupole-quadrupole interaction (4). Each of them is complementary to the Wigner's supermultiplet (2) (Fig.1) or the respective $S U_{\sigma}$ (2) on the right-hand side of the reduction scheme (Fig.2).

\section{Implications of the unified reduction schemes}

The unified reduction schemes, obtained in the previous subsection lead to the possibility to apply the presented approach in the description of real nuclear systems. These possibilities are based on the following results that are corollaries of the established correspondence between the respective subalgebras in the complementary chains, outlined by the oval shapes in Figs. 1 and 2.

\subsection{Relation between the basis states in the pairing and quadrupole limits}

From the above generalized reduction schemes it could be seen that the chains defining the reductions in them in Figs. 1 and 2 determine full-basis sets in which the basis states could be classified and the correspondence between the representations labeling them in the pairing and rotational bases could be explicitly obtained.

We present for illustration in Tables 1 and 2 the complete classification of the states in the simplest case of two particles along the reduction chains of the two reduction schemes in Figs. 1 and 2.

As we choose to expand the states of the pairing bases $\left|\Psi_{P t}\right\rangle$ (13), $\left|\Psi_{\text {Pisc }}\right\rangle$ (16) and $\left|\Psi_{P i v}\right\rangle$ (17), labeled by the set of numbers $\{i\}$, denoting the specific pairing labels $\{v[p], \beta\}$, $\left\{v_{S}, t_{S}, \beta\right\}$ and $\left\{v_{T}, t_{T}, \beta\right\}$ correspondingly through the set of rotational basis states (6) with the quantum numbers $\{\alpha(\lambda, \mu) K\}$ denoted as the set $\{j\}$ :

$$
\left|\Psi_{P}\right\rangle_{i} \equiv|\{f\}, i, L M\rangle=\sum_{j} C_{i j}|\{f\}, j, L M\rangle .
$$

As a result of the dynamical symmetry, the pairing interactions (8), (9) and (10) are diagonal in the pairing basises
(13), (16) and (17) with eigenvalues given in [17]. Using the expansions (18) in the $S U(3)$ basis states and the diagonalization procedure for its matrix in the $S U(3)$ basis

$$
\begin{array}{r}
<\Psi_{P}\left|H_{\text {pair }}\right| \Psi_{P}>=E_{\text {pair }}(m, i,[P],(S T))= \\
\sum_{j k} C_{k i}^{*} C_{i j} . \delta_{k j \cdot k}<\Psi_{R}\left|H_{\text {pair }}\right| \Psi_{R}>_{j},
\end{array}
$$

we obtain numerically the probability $\left|C_{i j}\right|^{2}$ with which the states of the $S U(3)$ basis enter into the expansion of each of the pairing bases. In this way we actually calculate the transformation brackets between the two chains [20], which is of great use when calculating the matrix elements of different operators in each of the chains. Also, this expansion could help to evaluate the importance (weight) of the different $S U(3)$ - states, when we need to impose restrictions on the basis because of computational difficulties. The known relations of the $S U 3$ labels $(\lambda, \mu)$ and the $\beta, \gamma$ shape variables of the geometrical model can be used for the analysis of the deformations of the pairing states, expressed through the respective $S U(3)$ ones.

\subsection{Phases and phase transitions in the microscopic PQM}

As stated in the Introduction, the dynamical symmetries unified in the reduction schemes presented in Figs. 1 and 2 can be considered as different phases, corresponding to the different types of residual interactions [21], that are included in the microscopic PQM. The phase transitions according to this definition involve actually a change of the symmetry from one to another of the exact limits of the model. The degree of this change is measured in practice by introducing one or more control parameter(s) that give the weight of each of the limits' interactions in the correct description of the energy spectra of a given nucleus. In the case of considering only two interactions in the nuclear Hamiltonian we need only one control parameter $-1<x<1$, that is introduced in the following way:

$$
V_{\text {res }}=\frac{1}{2}(1-x) V_{1}+\frac{1}{2}(1+x) V_{2},
$$

where at $x=-1$ we have pure $V_{1}$ interaction and at $x=1$ the limiting case of pure $V_{2}$ interaction is realized. At $x=0$ we have both interactions mixed with their respective strengths. This allows us to investigate the influence of these residual interactions on the spectra in real nuclear systems, which will be presented in the talk of Dr. K.P. Drumev [22]. In it we consider as $V_{2}$ always the quadrupole-quadrupole interaction $V_{2}=\chi Q \cdot Q$.

In the case when only one of the pairing modes is involved then we have as $V_{1}$ one of the three pairing interactions, defined by (8),(9) or (10). In the case of the PQM Hamiltonian, where we take into account all three types of pairing interactions proton-proton, neutron-neutron and proton-neutron, accounted for by the isoscalar (9) and isovector (10) pairing terms in addition to the quadrupolequadrupole interaction, in the Hamiltonian there are three 
Table 1. The classification of the states of 2 particles in the $d s$ shell $(\Omega=6)$ according to the reduction scheme (1)

\begin{tabular}{|c|c|c|c|c|c|c|c|c|}
\hline $\begin{array}{c}U(6) \\
\{\tilde{f}\}\end{array}$ & $\begin{array}{c}S O(6) \\
{[\mu]}\end{array}$ & $\begin{array}{c}S O_{p}(6) \\
v[p]\end{array}$ & $\begin{array}{c}S U(3) \\
(\lambda, \mu)\end{array}$ & $K$ & $\begin{array}{c}S O_{L}(3) \\
L\end{array}$ & $\begin{array}{c}U_{S T}(4) \\
\{f\}\end{array}$ & $\begin{array}{c}S O_{P}(6) \\
{[P]}\end{array}$ & $\begin{array}{c}S U_{S}(2) \times S U_{T}(2) \\
(S T)\end{array}$ \\
\hline$\left\{1^{2}\right\}_{15}$ & {$\left[1^{2}\right]_{15}$} & $2[1]$ & $(2,1)_{15}$ & 1 & $1,2,3$ & $\{2\}_{10}$ & {$\left[1^{3}\right]_{10}$} & $\begin{array}{l}(0,0)_{1} \\
(1,1)_{9}\end{array}$ \\
\hline$\{2\}_{21}$ & $\begin{array}{c}{[0]_{1}} \\
{[2]_{20}}\end{array}$ & $\begin{array}{c}0[0] \\
2\left[1^{3}\right]\end{array}$ & $\begin{array}{l}(4,0)_{15} \\
(0,2)_{6}\end{array}$ & $\begin{array}{l}0 \\
0\end{array}$ & $\begin{array}{c}0,2,4 \\
0,2\end{array}$ & $\left\{1^{2}\right\}_{6}$ & {$[1]_{6}$} & $\begin{array}{l}(1,0)_{3} \\
(0,1)_{3}\end{array}$ \\
\hline
\end{tabular}

Table 2. The classification of the states of 2 particles in the $d s$ shell $(\Omega=6)$ according to the reduction scheme in Fig. 2 for the isovector pairing only. In the case of the isoscalar pairing only, one should exchange the place of the $S$ and $T$ labels in the Table.

\begin{tabular}{cccccc|cc}
\hline$U(12)$ & $S p(12)$ & $S O(6) \times S U_{S}(2)$ & $S U(3) \times S U_{S}(2)$ & & $S O_{L}(3)$ & $U_{T}(2)$ & $S U_{T}(2)$ \\
$\{\tilde{f}\}$ & $<\tilde{\mu}>$ & {$[\tilde{\mu}] \times S$} & $(\lambda, \mu) \times S$ & $K$ & $L$ & $\{f\}$ & $T$ \\
\hline$\left\{1^{2}\right\}_{66}$ & $<1^{2}>_{65}$ & {$[2]_{20} \times\{0\}_{1}$} & $(4,0)_{15} \times 0_{1}$ & 0 & $0,2,4$ & $\{1\}_{3}$ & $\{1\}_{3}$ \\
& & {$\left[1^{2}\right]_{15} \times\{1\}_{3}$} & $(2,1)_{15} \times 1_{3}$ & 1 & $1,2,3$ & & \\
& $<0>_{1}$ & {$[0]_{1} \times\{0\}_{1}$} & $(0,2)_{6} \times 0_{1}$ & 0 & 0,2 & & \\
\hline$\{2\}_{78}$ & $<2>_{78}$ & {$[2]_{20} \times\{1\}_{3}$} & $(4,0)_{15} \times 1_{3}$ & 0 & $0,2,4$ & $\left\{1^{2}\right\}_{1}$ & $\{0\}_{1}$ \\
& & {$[0]_{1} \times\{1\}_{3}$} & $(2,1)_{15} \times 0_{1}$ & 1 & $1,2,3$ & & \\
& & {$\left[1^{2}\right]_{15} \times\{0\}_{1}$} & $(0,2)_{6} \times 1_{3}$ & 0 & 0,2 & & \\
\hline
\end{tabular}

phenomenological parameters $G_{0}, G_{1}$ and $\chi$ - their respective strengths. The later are evaluated trough a minimization procedure in the comparison of the theoretical spectrum of a given nucleus to the experimental energies. In order to study the influence of each of them on the reproduction of the experimental data, we need to introduce the two control parameters $y, z$ and a scaling parameter $c$

$$
y=\frac{\chi}{\chi+G_{1}}, z=\frac{\chi+G_{1}}{\chi+G_{0}+G_{1}}, c=\chi+G_{0}+G_{1} .
$$

In terms of these control parameters the phase diagram of the PQM Hamiltonian

$$
V_{\text {res }}=y z Q \cdot Q-(1-z) y S^{\dagger} \cdot S-(1-y) z P^{\dagger} \cdot P
$$

is a triangle, where the three limiting cases at its vertices are defined by the conditions: $y=z=1$ for the $S U(3)$ limit, $y=1, z=0$ for the isoscalar case and $y=0, z=1$ for the isovector one. By means of their values we can evaluate the role of each of the three interactions in the description of the realistic nuclear spectra in each nucleus.

\section{Conclusions}

The algebraic structure of the shell-model algebra $U(4 \Omega)$ is investigated to obtain its reductions through the microscopic pairing algebras, containing isoscalar $(T=0, S=$ $1)$, isovector $(T=1, S=0)$ total pairing operators and Elliott's $S U(3)$ algebra. The four reduction chains appear as distinct dynamical symmetries of the shell-model algebra, which allows the classification of the basis states of the system along each of them. Relations between these chains are established on the basis of their complementarity to the Wigner's spin-isospin $U_{S T}(4)$ symmetry or $S U_{T}(2) \sim S O_{T}(3), S U_{S}(2) \sim S O_{S}(3)$. The later allow for obtaining the transformation brackets with which the states of the $S U(3)$ basis enter into the expansion of the pairing bases. The obtained results elucidate the phases - dynamical symmetries of an extended Pairingplus-Quadrupole Model, realized in the framework of the Elliott's $S U(3)$ - scheme. The phase transitions between all these limits are studied by evaluating the weights of the different interactions in the PQM Hamiltonian for a particular nuclear system. The theory allows for a lot of applications to the real nuclear systems and can be used for interpretation of the experimental data and a systematic investigation of the development of nuclear collectivity trough the nuclear chart.

\section{Acknowledgements}

AIG is grateful for the support of the BLTP of JINR, Dubna, which made possible her participation in this conference. This work was also partially supported by the Bulgarian National Foundation for Scientific Research under Grant Number DFNIE02/6/12.12.2014.

\section{References}

[1] P.V. Isacker, Rep.Prog. Phys. 62, 1661 (1999); P. Van Isacker and K. Heyde, Exactly solvable models of nuclei, arXiv:1401.7512v1[nucl-th]

[2] S.T. Belyaev, Mat. Fys. Medd. Dan. Vid. Selsk. 31, 11 (1959)

[3] L.S. Kisslinger and R.A. Sorensen, Rev. Mod. Phys. 35, 853 (1963)

[4] M. Baranger and K. Kumar, Nucl. Phys. 62, 113 (1965)

[5] E.P. Wigner Phys. Rev. 51, 106 (1937)

[6] J.P. Draayer, in Algebraic Approaches to Nuclear Structure: Interacting Boson and Fermion Models, 423, Contemporary Concepts in Physics VI, ed. R.F. Casten (Harwood Academic, Chur, Switzerland, 1993)

[7] P.V. Isacker, Rep. Prog. Phys. 62, 1661 (1999) 
[8] V.K.B. Kota, Symmetries in Science XI, 265, eds. B. Gruber, G. Marmo, N. Yoshinaga, (Kluwer Academic Publishers, Dordrecht, 2004)

[9] J.P. Elliott, Proc. Roy. Soc. London, Ser. A245, 128 (1958); A245, 562 (1958)

[10] J.P. Draayer, Y. Leschber, S.C. Park, and R. Lopez, Comput. Phys. Commun. 56, 279 (1989)

[11] Y. Akiyama and J.P. Draayer, Comput. Phys. Commun. 5, 405 (1973)

[12] C. Bahri and J.P. Draayer, Comput. Phys. Commun. 83, 59 (1994)

[13] J.P. Draayer, Y. Leschber, S.C. Park, and R. Lopez, Comput. Phys. Commun. 56, 279 (1989)

[14] B.H. Flowers and S. Szpikowski, Proc. Phys. Soc. 84, 673 (1964)

[15] S.C. Pang, Nucl. Phys. A 128, 497 (1969)
[16] M. Moshinsky, C. Quesne, Phys. Lett. B 29, 482 (1969)

[17] V.K.B. Kota, Nucl. Phys. A 90, 321 (2006)

[18] V.V. Vanagas, Algebraic foundations of microscopic nuclear theory (Nauka, Moscow, 1988), (in russian)

[19] K.P. Drumev, A.I. Georgieva, NUCLEAR THEORY, 32, eds. A. Georgieva, N. Minkov (Heron Press, Sofia, 2013) p.151; Proceedings of the 32 Intern. Workshop on Nuclear Theory, (Rila Mountains, Bulgaria, June 24-29, 2013); http://ntl.inrne.bas.bg/workshop/2013/contributions/

[20] E. Santopinto, R. Bijker, and F. Iachello, J. Math. Phys. 37, 2674 (1996)

[21] J. Cseh, J. Phys.: Conf. Ser. 205, 012021 (2010)

[22] K.P. Drumev and A.I. Georgieva, "Interrelations between the pairing and quadrupole interactions in the microscopic shell model", in this volume. 